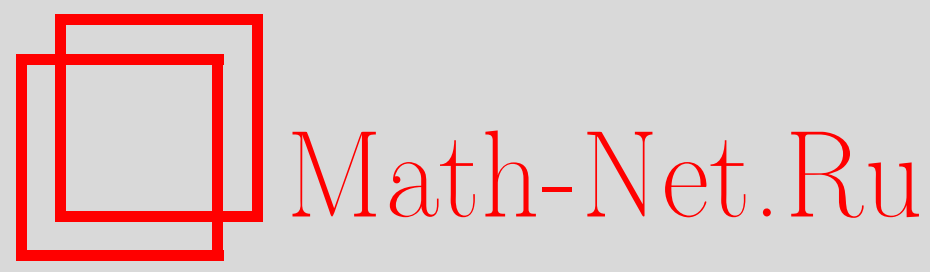

О. В. Камловский, Коэффициенты кросс-корреляции разрядных последовательностей равномерных линейных рекуррент над примарным кольцом вычетов, Матем. волр. криптогр., 2020, том 11, выпуск 1, 47-62

DOI: https://doi.org/10.4213/mvk314

Использование Общероссийского математического портала Math-Net.Ru подразумевает, что вы прочитали и согласны с пользовательским соглашением http://www.mathnet.ru/rus/agreement

Параметры загрузки:

IP: 54.162 .27 .143

26 апреля 2023 г., 09:24:44

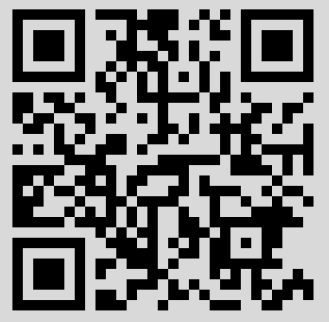


МАТЕМАТИЧЕСКИЕ ВОПРОСЫ КРИПТОГРАФИИ

2020 T. 11 № 1 C. 47-62

УДК $512.547+512.552$

DOI https://doi.org/10.4213/mvk314

\section{Коэффициенты кросс-корреляции разрядных последовательностей равномерных линейных рекуррент над примарным кольцом вычетов}

\section{О. В. Камловский}

ООО «Центр сертификационньх исследований», Москва

Получено 29.IV.2019

Аннотация. Изучается класс последовательностей элементов простого поля, полученных в результате выделения старшего p-ичного разряда из линейных рекуррентных последовательностей над кольцом вычетов по модулю $p^{n}$. Приводятся абсолютные и неабсолютные оценки сверху для модулей коэффициентов кросс-корреляции рассматриваемых последовательностей. Эти результаты позволяют указать условия, при которых отрезки разрядных последовательностей, полученных из различных исходных последовательностей, также являются различными.

Ключевые слова: равномерные последовательности, линейные рекуррентные последовательности, разрядные последовательности, коэффициенты кросс-корреляции

Cross-correlation coefficients for digit sequences of uniform linear recurrent sequences over the residue ring

\section{O. V. Kamlovskiy}

Certification Research Center, LLC, Moscow

Abstract. We study the class of sequences over the prime field with $p$ elements formed by the most significant $p$-ary digits of linear recurrent sequences over the residue ring modulo $p^{n}$. We obtain absolute and nonabsolute bounds for the cross-correlation coefficients of such sequences. This results lead to some sufficient conditions for segments of digit sequences obtained from different initial sequences to be different.

Keywords: uniform sequences, linear recurrent sequences, digit sequences, cross-correlation coefficients 


\section{Введение}

Линейные рекуррентные последовательности (ЛРП) над кольцами вычетов примарного порядка $p^{n}$, где $p-$ простое число, исследовались в ряде работ (см., например, $[1,2])$. Одной из сложных современных задач является получение точных формул или оценок для частот появлений элементов на отрезках рассматриваемых последовательностей. В то же время важно уметь строить так называемые равномерные ЛРП, в которых на отрезках длины, кратной периоду последовательности, каждый элемент кольца появляется одинаково часто (см. [3-9]). Кроме того, для практических приложений необходимо усложнять исходную последовательность, чтобы получать последовательности с большим рангом (линейной сложностью). Один из таких способов построения равномерных ЛРП был предложен А.С. Кузьминым и А.А. Нечаевым в [1]. Он основан на сложении исходной (основной) ЛРП со счетчиковой последовательностью и последующим выделением старшего $p$-ичного разряда. Частотные характеристики $r$-грамм на циклах и отрезках таких последовательностей были изучены в $[10,11]$. В частности, были получены оценки для модуля кросс-корреляционной функции, позволяющие гарантировать отсутствие сокращения периода и одинаковых последовательностей при различных начальных векторах ЛРП.

Данная работа продолжает исследования, начатые в [10,11]. В ней рассматриваются коэффициенты кросс-корреляции, характеризующие «близость» друг к другу отрезков исследуемых последовательностей. Отметим, что детально изучены сложные законы распределения элементов в основных последовательностях и их разрядных последовательностях (см., например, [2,12-16]). Целью данной работы является получение для коэффициентов кросс-корреляции разрядных последовательностей равномерных ЛРП результатов, аналогичных тем, которые известны для разрядных последовательностей основных ЛРП.

\section{1. Класс исследуемых последовательностей}

Пусть $R=\mathbb{Z}_{p^{n}}=\left\{0,1, \ldots, p^{n}-1\right\}-$ примарное кольцо вычетов. Рассмотрим унитарный реверсивный многочлен Галуа $F(x) \in R[x]$ (старший коэффициент многочлена $F(x)$ равен 1 , многочлен $\bar{F}(x)$, полученный из $F(x)$ приведением всех его коэффициентов по модулю $p$, неприводим над полем $P=\mathbb{Z}_{p}$ и $\left.\bar{F}(x) \neq x\right)$ степени $\operatorname{deg} F(x)=m-2$. Обозначим через $\oplus, \ominus, \otimes$ операции сложения, вычитания и умножения в поле $P$ соответственно. Пусть 


$$
H(x)=(x-1)^{2} F(x) .
$$

Будет рассматривать семейство $L_{R}(H)$ всех ЛРП над кольцом $R$ с характеристическим многочленом $H(x)$. Всюду далее будет изучаться случай, когда $\bar{F}(x) \neq x \ominus 1$. В этой ситуации (см. $[1,10])$ каждая ЛРП $\omega \in L_{R}(H)$ однозначно представима в виде

$$
\omega=v+u,
$$

где $v \in L_{R}\left((x-1)^{2}\right), u \in L_{R}(F)$, причем существуют такие однозначно определенные элементы $a, b \in R$, что

$$
v(i)=a i+b, \quad i \geqslant 0 .
$$

Последовательность $\omega$ является равномерной (см. $[1,10])$ тогда и только тогда, когда $a \in R^{*}$, где $R^{*}$ - мультипликативная группа кольца $R$. В этом случае ее период связан с периодом многочлена $\bar{F}(x)$ соотношением

$$
T(\omega)=p^{n} T(\bar{F})=\frac{p^{n}\left(p^{m-2}-1\right)}{d},
$$

где $d-$ некоторый делитель числа $p^{m-2}-1$.

Рассмотрим отображение $f: R \rightarrow P$, ставящее в соответствие каждому элементу $x \in R$ с $p$-ичным представлением

$$
x=x_{0}+p x_{1}+\ldots+p^{n-1} x_{n-1},
$$

где $x_{0}, x_{1}, \ldots, x_{n-1} \in P$, его старший разряд $x_{n-1}$. Обозначим через $\omega^{\prime}=f(\omega)$ последовательность с элементами $f(\omega(i))$, где $i \geqslant 0$. Учитывая равенство (2), получим

$$
\omega^{\prime}(i)=f(v(i)+u(i))=f(a i+b+u(i)), \quad i \geqslant 0 .
$$

Если $\omega-$ равномерная последовательность над кольцом $R$ (т. е. $a \in R^{*}$ ), то $\omega^{\prime}$ является равномерной последовательностью над полем $P$. Исследования работ $[1,11]$ показывают, что последовательности $\omega^{\prime}$ представляют интерес для криптографических приложений.

\section{2. Коэффициенты кросс-корреляции}

Пусть $\omega_{1}, \omega_{2} \in L_{R}(H)$ и выполнены равенства

$$
\omega_{1}(i)=a_{1} i+b_{1}+u_{1}(i), \quad \omega_{2}(i)=a_{2} i+b_{2}+u_{2}(i), \quad i \geqslant 0,
$$

где $a_{1}, a_{2}, b_{1}, b_{2} \in R, u_{1}, u_{2} \in L_{R}(F)$. Каждый гомоморфизм $\psi$ группы $(P, \oplus)$ в мультипликативную группу $\mathbb{C}^{*}$ поля комплексных чисел имеет вид (см. [17])

$$
\psi(x)=e^{2 \pi i \frac{c \otimes x}{p}}, \quad x \in P,
$$


где $c \in P$. Будем использовать обозначение $\psi=\psi_{c}$. Определим коэффициент кросс-корреляции последовательностей

$$
\omega_{1}^{\prime}=f\left(\omega_{1}\right), \quad \omega_{2}^{\prime}=f\left(\omega_{2}\right)
$$

равенством

$$
C_{\omega_{1}^{\prime}, \omega_{2}^{\prime}}(\psi, l, t)=\sum_{i=0}^{l-1} \psi\left(\omega_{1}^{\prime}(i) \ominus \omega_{2}^{\prime}(i+t)\right),
$$

где $l, t \in \mathbb{N}$. Так как всюду в дальнейшем результаты не будут зависеть от выбора элемента $c \in P, c \neq 0$, то для рассматриваемого коэффициента будем использовать обозначение $C_{\omega_{1}^{\prime}, \omega_{2}^{\prime}}(l, t)$. Значение величины $C_{\omega_{1}^{\prime}, \omega_{2}^{\prime}}(l, t)$ тесно связано с близостью между векторами

$$
\left(\omega_{1}^{\prime}(0), \ldots, \omega_{1}^{\prime}(l-1)\right), \quad\left(\omega_{2}^{\prime}(t), \ldots, \omega_{2}^{\prime}(l+t-1)\right) .
$$

Чем меньше величина $\left|C_{\omega_{1}^{\prime} \omega_{2}^{\prime}}(l, t)\right|$, тем заметнее отличаются друг от друга рассматриваемые векторы. В случае $p=2$ имеет место равенство

$$
C_{\omega_{1}^{\prime}, \omega_{2}^{\prime}}(l, t)=\sum_{i=0}^{l-1}(-1)^{\omega_{1}^{\prime}(i) \oplus \omega_{2}^{\prime}(i+t)},
$$

и несложно проверить, что расстояние $\rho_{\omega_{1}^{\prime}, \omega_{2}^{\prime}}(l, t)$ Хэмминга между векторами (9) вычисляется по формуле

$$
\rho_{\omega_{1}^{\prime}, \omega_{2}^{\prime}}(l, t)=\frac{l-C_{\omega_{1}^{\prime}, \omega_{2}^{\prime}}(l, t)}{2} .
$$

\section{3. Сведение к суммам характеров кольца вычетов}

Рассмотрим отображение $g=g_{c}: R \rightarrow \mathbb{C}$, определенное по правилу

$$
g_{c}(x)=\psi(f(x)), \quad x \in R,
$$

где $f$ - функция выделения старшего $p$-ичного разряда, $c \in R, c \neq 0$, а $\psi=\psi_{c}$. Обозначим через $L$ множество всех отображений из $R$ в $\mathbb{C}$. Относительно операций сложения и умножения на комплексные числа $L$ образует линейное пространство со скалярным произведением $S$, задаваемым для $h_{1}, h_{2} \in L$ по правилу

$$
S\left(h_{1}, h_{2}\right)=\frac{1}{|R|} \sum_{x \in R} h_{1}(x) \bar{h}_{2}(x),
$$


где черта обозначает комплексное сопряжение. Множество характеров группы $(R,+)$ состоит из характеров $\chi_{a}, a \in R$, определенных равенством (см., например, [17])

$$
\chi_{a}(x)=e^{2 \pi i \frac{a x}{p^{n}}}, \quad x \in R .
$$

Система характеров $\left\{\chi_{a}, a \in R\right\}$ образует ортонормированный базис евклидова пространства $(L, S)$, и справедливы равенства

$$
\begin{gathered}
g_{c}(x)=\sum_{d \in R} \nu_{c, d} \chi_{d}(x), \\
\nu_{c, d}=\frac{1}{|R|} \sum_{x \in R} g_{c}(x) \bar{\chi}_{d}(x) .
\end{gathered}
$$

В дальнейшем нам понадобится оценка величины

$$
\sigma\left(g_{c}\right)=\sum_{d \in R}\left|\nu_{c, d}\right|
$$

Лемма 1 ([12]). 1) $\nu_{c, d}=0$ при всех $d \in p R$, 2) $n$ ри $n \geqslant 2$

$$
\sigma\left(g_{c}\right)<\frac{2(n-1)}{\pi} \ln p+\frac{13}{40} p+\frac{7}{20} .
$$

Докажем результаты, позволяющие свести изучение коэффициента $C_{\omega_{1}^{\prime}, \omega_{2}^{\prime}}(l, t)$ к исследованию сумм значений аддитивных характеров кольца $R$.

Утверждение 1. Пусть $n \geqslant 2$, тогда

$$
\left|C_{\omega_{1}^{\prime}, \omega_{2}^{\prime}}(l, t)\right|<\left(\frac{2(n-1)}{\pi} \ln p+\frac{13}{40} p+\frac{7}{20}\right)^{2} \max _{d, r \in R^{*}}\left|\sum_{i=0}^{l-1} \chi\left(d \omega_{1}(i)+r \omega_{2}(i+t)\right)\right|,
$$

где $\chi=\chi_{1}$.

Доказательство. С использованием (8) и (11) получим

$$
\begin{gathered}
C_{\omega_{1}^{\prime}, \omega_{2}^{\prime}}(l, t)=\sum_{i=0}^{l-1} \psi_{c}\left(\omega_{1}^{\prime}(i)\right) \psi_{p \ominus c}\left(\omega_{2}^{\prime}(i+t)\right) \\
=\sum_{i=0}^{l-1} \psi_{c}\left(f\left(\omega_{1}(i)\right)\right) \psi_{p \ominus c}\left(f\left(\omega_{2}(i+t)\right)\right)=\sum_{i=0}^{l-1} g_{c}\left(\omega_{1}(i)\right) g_{p \ominus c}\left(\omega_{2}(i+t)\right)
\end{gathered}
$$




$$
\begin{aligned}
& =\sum_{i=0}^{l-1} \sum_{d \in R} \nu_{c, d} \chi_{d}\left(\omega_{1}(i)\right) \sum_{r \in R} \nu_{p \ominus c, r} \chi_{r}\left(\omega_{2}(i+t)\right) \\
& =\sum_{d, r \in R} \nu_{c, d} \nu_{p \ominus c, r} \sum_{i=0}^{l-1} \chi_{d}\left(\omega_{1}(i)\right) \chi_{r}\left(\omega_{2}(i+t)\right) .
\end{aligned}
$$

Согласно лемме 1 в этой формуле достаточно ограничиться суммированием по всем $d, r$ из множества $R \backslash p R=R^{*}$, поэтому

$$
C_{\omega_{1}^{\prime}, \omega_{2}^{\prime}}(l, t)=\sum_{d, r \in R^{*}} \nu_{c, d} \nu_{p \ominus c, r} \sum_{i=0}^{l-1} \chi\left(d \omega_{1}(i)+r \omega_{2}(i+t)\right)
$$

и, переходя к абсолютным величинам, получим

$$
\left|C_{\omega_{1}^{\prime}, \omega_{2}^{\prime}}(l, t)\right| \leqslant\left(\sum_{d \in R^{*}}\left|\nu_{c, d}\right|\right)\left(\sum_{r \in R^{*}}\left|\nu_{p \ominus c, r}\right|\right) \max _{d, r \in R^{*}}\left|\sum_{i=0}^{l-1} \chi\left(d \omega_{1}(i)+r \omega_{2}(i+t)\right)\right| .
$$

Для завершения доказательства утверждения 1 остается воспользоваться оценкой величины $\sigma\left(g_{c}\right)$ из леммы 1.

\section{4. Абсолютная оценка}

Получим оценку коэффициента кросс-корреляции (8), которая не будет зависеть от длины $l$ рассматриваемых отрезков ЛРП. Назовем такую оценку абсолютной.

Нам понадобится следующий вспомогательный результат.

Лемма 2 ([10]). Если $F(x)$ - реверсивный многочлен Галуа степени $m-2$ над кольцом $R=\mathbb{Z}_{p^{n}}, \bar{F}(x) \neq x \ominus 1$, то для каждой ЛРП $\omega \in L_{R}(H) \backslash L_{R}(x-1)$ и любого такого $l \in \mathbb{N}$, ито $l \leqslant p^{n} T(\bar{F})$,

$$
\left|\sum_{i=0}^{l-1} \chi(\omega(i))\right|<\left(\frac{4}{\pi^{2}} \ln T(\bar{F})+\frac{9}{5}\right) p^{\frac{m}{2}+2 n-2} .
$$

На множестве всех векторов-строк фиксированной длины над кольцом $R$ зададим бинарное отношение $\sim$, положив $\vec{\alpha} \sim \vec{\beta}$, если существует такой элемент $c \in R^{*}$, что $\vec{\beta}=c \vec{\alpha}$.

Теорема 1. Пусть последовательности $\omega_{1}, \omega_{2}$ определень равенством (5), $\omega_{1}^{\prime}, \omega_{2}^{\prime}$ заданы соотношением (7), F(x) - реверсивный 
многочлен Галуа степени $m-2$ над кольцом $R, \bar{F}(x) \neq x \ominus 1$ u $\left(u_{1}(0), \ldots, u_{1}(m-3), a_{1}\right) \nsim\left(u_{2}(t), \ldots, u_{2}(t+m-3), a_{2}\right), n \geqslant 2$. Тогда при всех $l \leqslant p^{n} T(\bar{F})$ для коэфбичиента кросс-коррелячии (8) справедлива оченка

$$
\left|C_{\omega_{1}^{\prime}, \omega_{2}^{\prime}}(l, t)\right|<\left(\frac{2(n-1)}{\pi} \ln p+\frac{13}{40} p+\frac{7}{20}\right)^{2}\left(\frac{4}{\pi^{2}} \ln T(\bar{F})+\frac{9}{5}\right) p^{\frac{m}{2}+2 n-2} .
$$

Доказательство. Покажем, что при условии

$$
\left(u_{1}(0), \ldots, u_{1}(m-3), a_{1}\right) \nsim\left(u_{2}(t), \ldots, u_{2}(t+m-3), a_{2}\right)
$$

последовательность с элементами $d \omega_{1}(i)+r \omega_{2}(i+t)$, где $i \geqslant 0$, не принадлежит множеству $L_{R}(x-1)$ при всех $d, r \in R^{*}$. Действительно, согласно $(5) d \omega_{1}(i)+r \omega_{2}(i+t)=$ const для всех $i \geqslant 0$ только если $\left(d a_{1}+r a_{2}\right) i+d u_{1}(i)+r u_{2}(i+t)=$ const для всех $i \geqslant 0$. В силу единственности представления (2) это равносильно тому, что $d a_{1}+r a_{2}=0$ и $d u_{1}(i)+r u_{2}(i+t)=0$ для всех $i \geqslant 0$, т. е. условию

$$
d\left(u_{1}(0), \ldots, u_{1}(m-3), a_{1}\right)+r\left(u_{2}(t), \ldots, u_{2}(t+m-3), a_{2}\right)=(0, \ldots, 0) .
$$

Для завершения доказательства остается воспользоваться леммой 2 и утверждением 1 .

\section{5. Сравнение с основными ЛРП}

Пусть $G(x)$ - унитарный реверсивный многочлен Галуа степени $m$ над кольцом $R=\mathbb{Z}_{p^{n}}, \delta_{1}, \delta_{2} \in L_{R}(G)-Л Р П$ и $\delta_{1}^{\prime}=f\left(\delta_{1}\right), \delta_{2}^{\prime}=f\left(\delta_{2}\right)-$ их $p$-ичные разрядные последовательности. Последовательности $\delta_{1}^{\prime}$ и $\delta_{2}^{\prime}$ зачастую рассматриваются как усложнения последовательностей $\delta_{1}$ и $\delta_{2}$ соответственно (см. $\left.[1,2]\right)$. Оценим коэффициент кросс-корреляции

$$
C_{\delta_{1}^{\prime}, \delta_{2}^{\prime}}(l, t)=\sum_{i=0}^{l-1} \psi\left(\delta_{1}^{\prime}(i) \ominus \delta_{2}^{\prime}(i+t)\right) .
$$

Теорема 2. Пусть $\delta_{1}, \delta_{2} \in L_{R}(G), G(x)$ - реверсивный многочлен Галуа степени $m$ над кольщом $R, \delta_{1}^{\prime}=f\left(\delta_{1}\right), \delta_{2}^{\prime}=f\left(\delta_{2}\right)$, $\left(\delta_{1}(0), \ldots, \delta_{1}(m-1)\right) \nsim\left(\delta_{2}(t), \ldots, \delta_{2}(t+m-1)\right), n \geqslant 2$. Тогда при всех $l \leqslant T(G)$

$$
\left|C_{\delta_{1}^{\prime}, \delta_{2}^{\prime}}(l, t)\right|<\left(\frac{2(n-1)}{\pi} \ln p+\frac{13}{40} p+\frac{7}{20}\right)^{2}\left(\frac{4}{\pi^{2}} \ln T(\bar{G})+\frac{9}{5}\right) p^{\frac{m}{2}+2 n-2} .
$$


Доказательство проводится аналогично доказательству теоремы 1 с использованием оценки (см. [12]).

$$
\left|\sum_{i=0}^{l-1} \chi(\delta(i))\right|<\left(\frac{4}{\pi^{2}} \ln T(\bar{G})+\frac{9}{5}\right) p^{\frac{m}{2}+2 n-2},
$$

справедливой для всех $l \leqslant T(G)$ и каждой ненулевой ЛРП $\delta \in L_{R}(G)$.

Теорема 2 обобщает на случай произвольного значения $p$ результаты работ $[13,14,16]$. Кроме того, при $p=2$ теорема 2 устанавливает несколько более точные оценки, чем результаты, приведенные в указанных статьях.

Отметим, что при фиксированных значениях $n, p$ и $m \rightarrow \infty$ правые части оценок из теорем 1 и 2 имеют вид $O\left(m p^{\frac{m}{2}}\right)$.

Рассмотрим оценки из теорем 1 и 2 в наиболее интересном частном случае, когда $F(x)$ и $G(x)$ - многочлены максимальных периодов: $T(F)=p^{n-1}\left(p^{m-2}-1\right), T(G)=p^{n-1}\left(p^{m}-1\right)$. В этом случае для равномерных ЛРП $\omega_{1}^{\prime}, \omega_{2}^{\prime}$ удается гарантировать несколько более точные оценки, чем для ЛРП $\delta_{1}^{\prime}, \delta_{2}^{\prime}$. Отметим, что в общем случае нельзя добиться равномерности последовательностей $\delta_{1}^{\prime}$ и $\delta_{2}^{\prime}$, в то время как равномерность последовательностей $\omega_{1}^{\prime}$ и $\omega_{2}^{\prime}$ достигается выбором обратимых элементов $a_{1}$ и $a_{2}$ в равенстве (5). Таким образом, разрядные последовательности равномерных ЛРП $\omega_{1}, \omega_{2}$ с точки зрения обоснованности свойств распределений элементов на отрезках более интересны, чем разрядные последовательности основных ЛРП $\delta_{1}, \delta_{2}$.

\section{6. Неабсолютная оценка}

Получим верхнюю оценку модуля коэффициента кросскорреляции (8), которая зависит от длины $l$ рассматриваемых отрезков ЛРП. Для этого понадобится соответствующая оценка суммы

$$
\sigma_{l}(\omega)=\sum_{i=0}^{l-1} \chi(\omega(i))
$$

Утверждение 2. Если $F(x)$ - реверсивный многочлен Галуа степени $m-2$ над кольиом $R=\mathbb{Z}_{p^{n}}, \bar{F}(x) \neq x \ominus 1, T(F)=p^{\nu}\left(p^{m-2}-1\right) / d$, $T(\bar{F}) \geqslant p^{2 n} / 3$, то для каждой ЛРП $\omega \in L_{R}(H) \backslash L_{R}(x-1)$ и любого 
такого $l \in \mathbb{N}$, что $l<T(\bar{F})$, въполнено неравенство

$$
\left|\sigma_{l}(\omega)\right| \leqslant\left(\frac{3 p^{n} l}{d}\left(p^{m-2}+(l-1)\left(d p^{n-1}-1\right) p^{\frac{m}{2}-1}\right)\right)^{\frac{1}{3}}
$$

Доказательство. Пусть последовательность $\omega$ удовлетворяет соотношению (2). По условию либо $a \neq 0$, либо $u$ - ненулевая последовательность. Если $u$ - нулевая последовательность, то согласно [10, теорема 1] $\left|\sigma_{l}(\omega)\right|<p^{n}$. В этом случае доказываемое неравенство имеет место, так как $3 T(\bar{F}) \geqslant p^{2 n}$. Пусть теперь $u$ - ненулевая ЛРП. Так как $F(x)$ - реверсивный многочлен, то $\omega$ является чисто периодической последовательностью и $T(\omega) \geqslant T(u) \geqslant T(\bar{F})$. Пусть $v_{k}=x^{k} \omega, k \geqslant 0$, сдвиг последовательности $\omega$ на $k$ шагов влево. Тогда множество $P(\omega)$ всех сдвигов ЛРП $\omega$ имеет вид $P(\omega)=\left\{v_{0}, v_{1}, \ldots, v_{T(\omega)-1}\right\}$. Согласно [18, теорема 1] будет выполнено неравенство

$$
\left|\sigma_{l}(\omega)\right| \leqslant\left(3 \sum_{v \in P(\omega)}\left|\sigma_{l}(v)\right|^{2}\right)^{\frac{1}{3}}
$$

Период $T(\omega)$ является делителем числа $p^{n} T(\bar{F})$, поэтому справедливо соотношение

$$
\sum_{v \in P(\omega)}\left|\sigma_{l}(v)\right|^{2}=\frac{T(\omega)}{p^{n} T(\bar{F})} \sum_{k=0}^{p^{n} T(\bar{F})-1}\left|\sigma_{l}\left(v_{k}\right)\right|^{2}
$$

Оценим сверху величину

$$
R(\omega)=\sum_{k=0}^{p^{n} T(\bar{F})-1}\left|\sigma_{l}\left(v_{k}\right)\right|^{2}
$$

Имеет место равенство

$$
R(\omega)=\sum_{k=0}^{p^{n} T(\bar{F})-1} \sigma_{l}\left(v_{k}\right) \overline{\sigma_{l}\left(v_{k}\right)}
$$

где $\overline{\sigma_{l}\left(v_{k}\right)}$ - число, комплексно сопряженное к числу $\sigma_{l}\left(v_{k}\right)$. Тогда

$$
R(\omega)=\sum_{k=0}^{p^{n} T(\bar{F})-1} \sum_{i=0}^{l-1} \chi\left(v_{k}(i)\right) \sum_{j=0}^{l-1} \chi\left(-v_{k}(j)\right) .
$$


Из (16) следует, что

$$
\begin{aligned}
R(\omega)=\sum_{k=0}^{p^{n} T(\bar{F})-1} \sum_{i, j=0}^{l-1} \chi( & \left.v_{k}(i)-v_{k}(j)\right) \\
& =\sum_{k=0}^{p^{n} T(\bar{F})-1} \sum_{i, j=0}^{l-1} \chi(a i-a j+u(k+i)-u(k+j)) .
\end{aligned}
$$

Представим ЛРП и с использованием функции «след» (см., например, [1]):

$$
u(i)=\operatorname{Tr}_{R}^{S}\left(b \alpha^{i}\right), i \geqslant 0,
$$

где $S=G R\left(p^{(m-2) n}, p^{n}\right)$ - кольцо Галуа характеристики $p^{n}$, состоящее из $p^{(m-2) n}$ элементов, $\alpha$ - корень многочлена $F(x)$ в кольце $S$, а $b-$ ненулевой элемент кольца $S$. Тогда

$$
R(\omega)=\sum_{i, j=0}^{l-1} \chi(a(i-j)) \sum_{k=0}^{p^{n} T(\bar{F})-1} \chi^{\prime}\left(b\left(\alpha^{i}-\alpha^{j}\right) \alpha^{k}\right)
$$

где $\chi^{\prime}$ - аддитивный характер кольца $S$, определенный равенством $\chi^{\prime}(y)=\chi\left(\operatorname{Tr}_{R}^{S}(y)\right)$. Выделив отдельно слагаемое, соответствующее случаю $i=j$, получим

$$
R(\omega)=p^{n} T(\bar{F}) l+p^{n-\nu} \sum_{i \neq j} \chi(a(i-j)) \sum_{k=0}^{p^{\nu} T(\bar{F})-1} \chi^{\prime}\left(b\left(\alpha^{i}-\alpha^{j}\right) \alpha^{k}\right) .
$$

Значит,

$$
\begin{gathered}
R(\omega)=p^{n} T(\bar{F}) l \\
+p^{n-\nu} \sum_{i \neq j} \chi(a(i-j))\left(\sum_{k=0}^{p^{\nu} T(\bar{F})-1} \chi^{\prime}\left(b\left(\alpha^{i}-\alpha^{j}\right) \alpha^{k}\right)+\frac{p^{\nu}}{d}\right)-\frac{p^{n}}{d} \sum_{i \neq j} \chi(a(i-j)) .
\end{gathered}
$$

Если $a=0$, то

$$
\sum_{i \neq j} \chi(a(i-j))=l(l-1)
$$

Если $a \neq 0$, то

$$
\sum_{i \neq j} \chi(a(i-j))=-l+\sum_{i, j=0}^{l-1} \chi(a(i-j))=-l+\left|\sum_{i=0}^{l-1} \chi(a i)\right|^{2} .
$$


Переходя к рассмотрению абсолютных величин, получим

$$
\begin{aligned}
|R(\omega)| & \leqslant p^{n} T(\bar{F}) l+\frac{l p^{n}}{d}+p^{n-\nu} l(l-1) \max _{i \neq j}\left|\sum_{k=0}^{p^{\nu} T(\bar{F})-1} \chi^{\prime}\left(b\left(\alpha^{i}-\alpha^{j}\right) \alpha^{k}\right)+\frac{p^{\nu}}{d}\right| \\
& =\frac{l p^{m+n-2}}{d}+p^{n-\nu} l(l-1) \max _{i \neq j}\left|\sum_{k=0}^{p^{\nu} T(\bar{F})-1} \chi^{\prime}\left(b\left(\alpha^{i}-\alpha^{j}\right) \alpha^{k}\right)+\frac{p^{\nu}}{d}\right| .
\end{aligned}
$$

Заметим, что образ элемента $\alpha^{i}-\alpha^{j}$ при действии естественного эпиморфизма колец $S \rightarrow S / p S$ равен $\bar{\alpha}^{i}-\bar{\alpha}^{j}$, где $\bar{\alpha}-$ корень многочлена $\bar{F}(x)$ в поле $G F\left(q^{m-2}\right)$. Тогда при всех различных $i, j \in \overline{0, l-1}$ и $l \leqslant T(\bar{F})$ элемент $\bar{\alpha}^{i}-\bar{\alpha}^{j}$ ненулевой, а значит, элемент $\alpha^{i}-\alpha^{j}-$ обратимый элемент кольца $S$. С использованием оценки из [19] получим

$$
\left|\sum_{k=0}^{p^{\nu} T(\bar{F})-1} \chi^{\prime}\left(b\left(\alpha^{i}-\alpha^{j}\right) \alpha^{k}\right)+\frac{p^{\nu}}{d}\right| \leqslant \frac{d p^{n-1}-1}{d} p^{\frac{m}{2}+\nu-1} .
$$

Таким образом,

$$
|R(\omega)| \leqslant \frac{l p^{n}}{d}\left(p^{m-2}+(l-1)\left(d p^{n-1}-1\right) p^{\frac{m}{2}-1}\right),
$$

а значит, согласно равенству (15)

$$
\sum_{v \in P(\omega)}\left|\sigma_{l}(v)\right|^{2} \leqslant \frac{l p^{n}}{d}\left(p^{m-2}+(l-1)\left(d p^{n-1}-1\right) p^{\frac{m}{2}-1}\right) .
$$

Для завершения доказательства остается воспользоваться неравенством (14).

Основным результатом этого раздела является следующая неабсолютная оценка модуля коэффициента кросс-корреляции.

Теорема 3. Пусть в условиях теоремы 1 дополнительно выполнены соотношения $T(\bar{F}) \geqslant p^{2 n} / 3, l<T(\bar{F})$, тогда

$$
\left|C_{\omega_{1}^{\prime}, \omega_{2}^{\prime}}(l, t)\right|<C(n, p)^{2}\left(\frac{3 p^{n} l}{d}\left(p^{m-2}+(l-1)\left(d p^{n-1}-1\right) p^{\frac{m}{2}-1}\right)\right)^{\frac{1}{3}},
$$

əде

$$
C(n, p)=\frac{2(n-1)}{\pi} \ln p+\frac{13}{40} p+\frac{7}{20}
$$


Доказательство. проводится аналогично доказательству теоремы 1 с использованием утверждений 1 и 2.

Покажем, что полученная неабсолютная оценка точнее абсолютной оценки из теоремы 1 при всех $l$, удовлетворяющих условиям

$$
p^{\frac{m}{2}-1} \leqslant l \leqslant \frac{1}{\sqrt{3}} C(F)^{\frac{3}{2}} p^{\frac{m}{2}+2 n-2},
$$

где

$$
C(F)=\frac{4}{\pi^{2}} \ln T(\bar{F})+\frac{9}{5} .
$$

Действительно, это так при условии

$$
\frac{3 p^{n} l}{d}\left(p^{m-2}+(l-1)\left(d p^{n-1}-1\right) p^{\frac{m}{2}-1}\right)<C(F)^{3} p^{\frac{3 m}{2}+6 n-6} .
$$

Последнее неравенство заведомо выполнено, если

$$
\frac{l}{d}\left(p^{m-2}+l\left(d p^{n-1}-1\right) p^{\frac{m}{2}-1}\right) \leqslant \frac{1}{3} C(F)^{3} p^{\frac{3 m}{2}+5 n-6} .
$$

Так как $l \geqslant p^{m / 2-1}$, то неравенство (18) выполняется при условии

$$
\frac{l}{d}\left(p^{\frac{m}{2}-1} l+l\left(d p^{n-1}-1\right) p^{\frac{m}{2}-1}\right) \leqslant \frac{1}{3} C(F)^{3} p^{\frac{3 m}{2}+5 n-6}
$$

что равносильно

$$
l^{2} \leqslant \frac{1}{3} C(F)^{3} p^{m+4 n-4} .
$$

\section{7. Различные отрезки последовательностей}

Рассмотрим вопрос о значении длины $l$, при которой отрезки (9) являются различными.

Теорема 4. Пусть последовательности $\omega_{1}, \omega_{2}$ определены равенством (5), последовательности $\omega_{1}^{\prime}, \omega_{2}^{\prime}$ задань соотношением (7), $F(x)$ - реверсивный многочлен Галуа степени $m-2$ над кольцом $R, m \geqslant 4, n \geqslant 2, \bar{F}(x) \neq x \ominus 1,\left(u_{1}(0), \ldots, u_{1}(m-3), a_{1}\right) \nsim\left(u_{2}(t)\right.$, $\left.\ldots, u_{2}(t+m-3), a_{2}\right)$,

$$
l_{0}=\left\lceil 3 C(n, p)^{6} p^{\frac{m}{2}+2 n-2}\right\rceil .
$$

Тогда если $l_{0}<T(\bar{F})$, то

$$
\left(\omega_{1}^{\prime}(0), \ldots, \omega_{1}^{\prime}\left(l_{0}-1\right)\right) \neq\left(\omega_{2}^{\prime}(t), \ldots, \omega_{2}^{\prime}\left(t+l_{0}-1\right)\right) .
$$


Доказательство. Допустим, что рассматриваемые отрезки последовательностей равны. Тогда $\left|C_{\omega_{1}^{\prime}, \omega_{2}^{\prime}}\left(l_{0}, t\right)\right|=l_{0}$. Так как $C(n, p) \geqslant 1$, $m \geqslant 4$, то из условия $T(\bar{F})>l_{0}$ следует, что $3 T(\bar{F}) \geqslant p^{2 n}$. Тогда по теореме 3

$$
l_{0}=\left|C_{\omega_{1}^{\prime}, \omega_{2}^{\prime}}\left(l_{0}, t\right)\right|<C(n, p)^{2}\left(\frac{3 p^{n} l_{0}}{d}\left(p^{m-2}+\left(l_{0}-1\right)\left(d p^{n-1}-1\right) p^{\frac{m}{2}-1}\right)\right)^{\frac{1}{3}} .
$$

Покажем, что при всех

$$
l \geqslant 3 C(n, p)^{6} p^{\frac{m}{2}+2 n-2}
$$

будет выполнено соотношение

$$
C(n, p)^{2}\left(\frac{3 p^{n} l}{d}\left(p^{m-2}+(l-1)\left(d p^{n-1}-1\right) p^{\frac{m}{2}-1}\right)\right)^{\frac{1}{3}} \leqslant l,
$$

которое будет противоречить соотношению (19) при подстановке значения $l=l_{0}$. Неравенство (20) имеет место, если

$$
\frac{3 p^{n} C(n, p)^{6}}{d}\left(p^{m-2}+l\left(d p^{n-1}-1\right) p^{\frac{m}{2}-1}\right) \leqslant l^{2} .
$$

Так как рассматривается случай $l \geqslant p^{m / 2-1}$, то полученное неравенство заведомо выполнено при условии

$$
\frac{3 p^{n} C(n, p)^{6}}{d}\left(l p^{\frac{m}{2}-1}+l\left(d p^{n-1}-1\right) p^{\frac{m}{2}-1}\right) \leqslant l^{2}
$$

т. е. при

$$
l \geqslant 3 C(n, p)^{6} p^{\frac{m}{2}+2 n-2}
$$

Теорема 4 показывает, что если $p, n$ фиксированы, а $m \rightarrow \infty$, то векторы (9) различаются при длине $l=O\left(p^{\frac{m}{2}}\right)$.

\section{8. Неабсолютная оценка для больших длин отрезков}

Оценка из теоремы 3 справедлива при $l<T(\bar{F})$, в то время как оценка из теоремы 1 имеет место при $l \leqslant p^{n} T(\bar{F})$. Предложим подход, позволяющий получить неабсолютные оценки на длине $l \leqslant p^{n}(T(\bar{F})-1)$. 
Теорема 5. Пусть в условиях теоремы 1 дополнительно выполнено соотношение $l \leqslant p^{n}(T(\bar{F})-1)$, тогда

$$
\left|C_{\omega_{1}^{\prime}, \omega_{2}^{\prime}}(l, t)\right|<p^{n} C(n, p)^{2}\left(\frac{3 l_{1}}{d}\left(p^{m-2}-l_{1}+\left(l_{1}-1\right)\left(d p^{n-1}-1\right) p^{\frac{m}{2}-1}\right)\right)^{\frac{1}{3}},
$$

где $l_{1}=\left\lfloor\frac{l-1}{p^{n}}\right\rfloor+1$.

Доказательство. Из утверждения 1 следует, что

$$
\left|C_{\omega_{1}^{\prime}, \omega_{2}^{\prime}}(l, t)\right|<C(n, p)^{2} \max _{\omega \in L_{R}(H) \backslash L_{R}(x-1)}\left|\sum_{i=0}^{l-1} \omega(i)\right| .
$$

Согласно [10] если $\omega$ имеет вид (2), где $u$ - нулевая последовательность, то

$$
\left|\sum_{i=0}^{l-1} \omega(i)\right|<p^{n}
$$

а если $u$ - ненулевая последовательность, то

$$
\left|\sum_{i=0}^{l-1} \omega(i)\right| \leqslant \sum_{j=1}^{p^{n}}\left|\sum_{k=0}^{l_{j}-1} v_{j}(k)\right|
$$

где $l_{j}=\left\lfloor\frac{l-j}{p^{n}}\right\rfloor+1$, а $v_{j}(k)=u\left(j-1+k p^{n}\right), k \geqslant 0$. При этом последовательность $v_{j}$ является ненулевой ЛРП, характеристический многочлен которой - реверсивный многочлен Галуа степени $m-2$ и периода $\left(p^{m-2}-1\right) / d$. Из [18, следствие 1] следует, что при $l_{j}<T(\bar{F})$ справедлива оценка

$$
\left|\sum_{k=0}^{l_{j}-1} v_{j}(k)\right| \leqslant\left(\frac{3 l_{j}}{d}\left(p^{m-2}-l_{j}+\left(l_{j}-1\right)\left(d p^{n-1}-1\right) p^{\frac{m}{2}-1}\right)\right)^{\frac{1}{3}} .
$$

Заметим, что правая часть последнего неравенства возрастает с ростом $l_{j}$ и всегда больше либо равна 1 . Кроме того, по условию

$$
l_{1}=\left\lfloor\frac{l-1}{p^{n}}\right\rfloor+1=\frac{l-a}{p^{n}}+1<\frac{l}{p^{n}}+1 \leqslant T(\bar{F}),
$$

где $a \in\left\{1, \ldots, p^{n}\right\}$ выбрано так, что $p^{n}$ делит $l-a$. Таким образом, 


$$
\left|\sum_{i=0}^{l-1} \omega(i)\right| \leqslant p^{n}\left(\frac{3 l_{1}}{d}\left(p^{m-2}-l_{1}+\left(l_{1}-1\right)\left(d p^{n-1}-1\right) p^{\frac{m}{2}-1}\right)\right)^{\frac{1}{3}}
$$

и остается воспользоваться неравенством (21).

Оценка из теоремы 5 оказывается несколько менее точной, чем оценка из теоремы 3, однако она представляет интерес, если длина отрезков $l \geqslant T(\bar{F})$, так как в этом случае теорема 3 неприменима. По аналогии с вышеприведенными рассуждениями можно показать, что оценка теоремы 5 заведомо точнее абсолютной оценки из теоремы 1, если

$$
p^{\frac{m}{2}-1} \leqslant l_{1} \leqslant \frac{1}{\sqrt{3}} C(F)^{\frac{3}{2}} p^{\frac{m}{2}+2 n-2} .
$$

Результаты работы показывают, что разрядные последовательности равномерных ЛРП являются усложнениями исходных ЛРП и могут быть использованы при построении псевдослучайных чисел.

\section{Список литературы}

[1] Kurakin V. L., Kuzmin A. S., Mikhalev A. V., Nechaev A. A., "Linear recurring sequences over rings and modules", J. Math. Sci., 76:6 (1995), 2793-2915.

[2] Кузьмин А.С., Куракин В.Л., Нечаев А.А., "Псевдослучайные и полилинейные последовательности", Труды по дискретной математике, 1 (1997), 139-202.

[3] Кейперс Л., Нидеррайтер Г., Равномерное распределение последовательностей, М.: Наука, 1985, 408 с.

[4] Ларин М.В., “Транзитивные полиномиальные преобразования колец вычетов”, Дискретная математика, 14:2 (2002), 20-32.

[5] Анашин В.С., "Равномерно распределенные последовательности целых $p$-адических чисел", Дискретная математика, 14:4 (2002), 3-64.

[6] Herendi T., "Uniform distribution of linear recurring sequences modulo prime powers", Finite Fields and Appl., 10:1 (2004), 1-23.

[7] Knight M.J., Webb W.A., "Uniform distribution of third order linear recurrence sequences", Acta Arith, 36 (1980), 7-20.

[8] Narkiewicz W., "Uniform distribution of sequences of integers in residue classes", Lect. Notes Math., 1087, 1984, 140 pp.

[9] Turnwald G., "Uniform distribution of second-order linear recurring sequences", Proc. Amer. Math. Soc., 96 (1986), 189-198.

[10] Камловский О.В., "Распределение $r$-грамм в одном классе равномерных последовательностей над кольцами вычетов", Проблемы передачи информации, 50:1 (2014), 98115.

[11] Камловский О.В., "Равномерные последовательности над простыми полями, построенные из одного класса линейных рекуррент над кольцами вычетов”, Проблемы передачи информачии, 50:2 (2014), 60-76.

[12] Камловский О.В., "Частотные характеристики разрядных последовательностей линейных рекуррент над кольцами Галуа", Изв. РАН. Сер. матем., 77:6 (2013), 71-96. 
[13] Sole P., Zinoviev D., "Distribution of $r$-patterns in the most significant bit of a maximum length sequence over $\mathbb{Z}_{2^{l}}$ ", Lect. Notes Comput. Sci., 3486 (2005), 275-281.

[14] Sole P., Zinoviev D., "The most significant bit of maximum-length sequences over $\mathbb{Z}_{2^{l}}$ : autocorrelation and imbalance", IEEE Trans. Inf. Theory, 50:8 (2006), 1844-1846.

[15] Qi W., Zhou J., "Distribution of 0 and 1 in the highest level of primitive sequences over $\mathbb{Z}_{2} e$, Science in China (Ser. A), 40:6 (1997), 606-611.

[16] Hu H., Feng D., Wu W., "Incomplete exponential sums over Galois rings with applications to some binary sequences derived from $\mathbb{Z}_{2^{l}} "$, IEEE Trans. Inf. Theory, 52:5 (2006), $2260-2265$.

[17] Лидл Р., Нидеррайтер Г., Конечные поля, 1, 2, М.: Мир, 1988, 822 с.

[18] Камловский О.В., "Метод В.М. Сидельникова для оценки числа знаков на отрезках линейных рекуррентных последовательностей над кольцами Галуа", Maтем. заметки, 91:3 (2012), 371-382.

[19] Камловский О.В., Кузьмин А.С., "Оценки частот появления элементов в линейных рекуррентных последовательностях над кольцами Галуа", Фундам. и прикл. математика, 6:4 (2000), 1083-1094. 